\title{
Prototipo para medir la profundidad de polimerización de materiales dentales en la práctica odontológica
}

\author{
Prototype to measure the polymerization depth \\ of dental materials in the odontology practical
}

\author{
Ysabel Victoria Morales Alva*
}

http://dx.doi.org/10.21503/CienciayDesarrollo.2007.v8.04

\section{RESUMEN}

El objetivo de la investigación es diagnosticar y diseñar un prototipo para evaluar la profundidad de la fotopolimerización de los materiales dentales en la aplicación práctica de la odontología.

El tipo de investigación ha sido aplicativo, y el nivel, descriptivo-correlacional. La muestra estuvo constituida por 50 profesionales que laboran actualmente en el Hospital Luis Sáenz de la Policía Nacional del Perú. El diseño metodológico fue el descriptivo-correlacional.

Las técnicas empleadas para la recolección de datos fueron la observación directa, la revisión documental y la aplicación de una encuesta, cuyos datos fueron luego procesados, analizados e interpretados.

La conclusión general a la que se llegó fue la de que existe suficiente evidencia estadística para afirmar que con el prototipo para medir la profundidad de fotopolimerización se mejorarán las restauraciones dentales de los pacientes que acuden a los consultorios de odontología del Hospital Central Luis Sáenz de La Policía Nacional del Perú, como lo aseveran los odontólogos que laboran en dicha institución.

Palabras clave: biomateriales dentales, revivan compuestas, polimerización, materiales, fotocurado, práctica odontológica.

\section{ABSTRACT}

The aim (lens) of the investigation is to diagnose and to design a prototype to evaluate the depth of the photopolimerization of the dental materials in the practical application of the Odontology.

The type of investigation has been aplicativo and the level descriptivo the sample was constituted by 50 that nowadays work in the Hospital Luis Sáenz of the National Police of the Peru and the methodological design was the descriptive.

The technologies (skills) used for the compilation of information were: the direct observation, the documentary review and the application of a survey, which information was tried, analyzed and interpreted.

The general conclusion to which it (he, she) came: sufficient statistical evidence exists to affirm that with the prototype to measure the depth of photopolimerization will be improved the dental restorations of the patients who come to the doctor's offices of Odontolgy of the Central Hospital Luis Sáenz of The National Police of the Peru, since it is affirmed by the Odontologists who work in the above mentioned institution.

Key words: dental biomaterials, re-lives compound, polimerization, through materials, phototreated, odontology practice. 


\section{INTRODUCCIÓN}

En la actualidad, el acelerado avance en biomateriales dentales, específicamente de resinas compuestas, exige al facultativo tener la oportunidad de validar sus conocimientos en prácticas odontológicas sencillas, eficientes y con múltiples opciones para tratamientos cada vez más exigentes. Debemos tener presente que aún existen ciertas limitaciones en la parte clínica, como son la insuficiente adhesión a la dentina y el estrés superficial residual de las resinas polimerizadas.

Diversas investigaciones sobre los materiales de fotocurado realizadas hasta la actualidad nos han permitido evaluar las propiedades mecánicas de los biomateriales dentales, entre las cuales podemos mencionar la dureza del material polimerizado y la profundidad de polimerización, la cual es precisamente motivo de nuestro estudio. El objetivo es alcanzar un mejor manejo clínico de materiales restauradores, contribuyendo de esta manera al avance de nuestros conocimientos científicos de la materia.

Debido a que las opciones tecnológicas a la hora de elegir los adecuados materiales de fotocurado han aumentado en los últimos años, se hace imprescindible conocer los tipos y características básicas de los diferentes materiales dentales de fotocurado. De esta manera, será más fácil decidir la que se adapta mejor a nuestras necesidades en la práctica clínica.

El propósito del presente estudio es diseñar un prototipo para evaluar la profundidad de fotopolimerización de los materiales dentales, lo que además permitirá ampliar los conocimientos relacionados con la especialidad de odontología restauradora.

\section{MATERIAL Y MÉTODO}

\section{Tipo y nivel de investigación}

\section{Tipo de investigación}

Por la forma en que se ha planteado la investigación y por los objetivos de la misma, reúne las características de una investigación aplicativa, en razón de que para llevar a cabo dicho estudio se utilizaron conocimientos sobre cómo diseñar un prototipo para medir la profundidad de fotopolimerizacion de restauraciones dentales y poder aplicarlo al mejoramiento de la profundidad de fotopolimerización de las restauraciones dentarias.

\section{Nivel de investigación}

La investigación reúne las condiciones para ser considerada como descriptiva, correlacional y explicativa.

Es descriptiva porque en un primer momento describe el diseño del prototipo para medir la profundidad de fotopolimerización de restauraciones dentales. Es correlacional debido a la relación que existe entre las variables materia de la investigación. Y es explicativa porque muestra de manera clara y comprensible el uso del diseño del prototipo para medir la profundidad de fotopolimerización de restauraciones dentales y cómo puede ser aplicado para mejorar la profundidad de fotopolimerización de las restauraciones dentarias.

\section{Método de la investigación}

Se han aplicado métodos cuantitativos y cualitativos, así como la inducción el análisis y la síntesis.

\section{Técnicas de recolección de información}

Se han aplicado la observación directa, la revisión documental y una encuesta aplicada a odontólogos. Luego se ha empleado el modelo estadístico. 


\section{Empleo del modelo estadistico}

El modelo estadístico empleado guarda relación con la metodología científica para la recolección, organización, tabulación, presentación y análisis de datos. Esto nos ha permitido planear, analizar e interpretar los resultados de la investigación.

Las operaciones realizadas con los datos obtenidos a través de las diversas técnicas de recopilación de datos (observación directa, revisión documental y encuestas) han sido las siguientes:

a. Procesamiento de datos, es decir la organización de la información obtenida, la misma que ha posibilitado extraer conclusiones de sus análisis.

b. La codificación o asignación de símbolos o números a todas y cada una de las categorías de respuestas recogidas en la información.

c. La tabulación o proceso que permite determinar la frecuencia del fenómeno objeto de investigación.

d. La formación de tablas o cuadros que posibilita la agrupación de los datos en tablas o cuadros de doble entrada convenientemente preparados, facilitando así la aplicación de métodos estadísticos, especialmente de los elementos de la estadística descriptiva. Esto ha permitido analizar los estadígrafos de posición o tendencia central, como la media, la mediana y la moda.

El respectivo procesamiento se llevará a cabo mediante el paquete estadístico SPSS, versión 12.

\section{Población y muestra}

La población de estudio estuvo constituida por los 40 odontólogos que laboran en el Hospital Central Luis Sáenz de la Policía Nacional del Perú. Considerando esta cantidad, se estudió a todos los odontólogos que trabajan en este hospital.

\section{RESULTADOS DE LA INVESTIGACIÓN}

I. Resultados de la encuesta aplicada a los odontólogos

P1) Información general: estado civil

Estado civil

\begin{tabular}{|c|c|c|c|}
\hline \\
\hline & & Frecuencia & Porcentaje \\
\hline \multirow[t]{4}{*}{ Válidos } & Soltero & 15 & 37,5 \\
\hline & Casado & 20 & 50,0 \\
\hline & Concubinato & 5 & 12,5 \\
\hline & Total & 40 & 100,0 \\
\hline
\end{tabular}

La mitad de los encuestados señaló que eran casados, el $37,5 \%$ indicó que eran solteros, y el $12,5 \%$ que estaban en situación de concubinato.

P2) Información general: edad.

\section{Edad}

Frecuencia Porcentaje

\begin{tabular}{rrrr}
\hline Válidos & $22-30$ & 17 & 42,5 \\
& $31-50$ & 18 & 45,0 \\
& 51 - más & 5 & 12,5 \\
& Total & 40 & 100,0 \\
\hline
\end{tabular}

El 45\% de los encuestados tiene una edad que oscila entre los 31 y 50 años de edad, un $42,5 \%$ entre los 22 y 30 años (lo que indica una población relativamente joven), y sólo el 12,5\% tiene más de 51 años.

P3) Información general: sexo.

\begin{tabular}{clcc}
\multicolumn{5}{c}{ Sexo } \\
& & & \\
& & Frecuencia & Porcentaje \\
\hline Válidos & F & 18 & 45,0 \\
& M & 22 & 55,0 \\
& Total & 40 & 100,0 \\
\hline
\end{tabular}

El 55,0\% de los encuestados son de sexo masculino, y el $45,0 \%$ son de sexo femenino. 
P4) Información general: tiempo que ejerce la profesión.

Tiempo que ejerce la profesión

Frecuencia Porcentaje

\begin{tabular}{rlrr}
\hline Válidos & 2 - 7 años & 17 & 42,5 \\
& $8-12$ años & 18 & 45,0 \\
12 - más & 5 & 12,5 \\
Total & 40 & 100,0 \\
\hline
\end{tabular}

El 45,0 \% viene ejerciendo la profesión entre 8 y 12 años, lo que acredita experiencia, mientras que el $42,5 \%$ oscila entre los 2 y 7 años, mientras que el 12,5\% tiene más de 12 años en el ejercicio profesional. Consideramos importante esta información porque los jóvenes profesionales son los más inclinados a introducir metodologías y técnicas nuevas en el trabajo profesional.

\section{Preguntas}

\section{Variable dependiente: profundidad de polimerización}

P5) ¿Puede usted calcular la profundidad de las restauraciones?

¿Puede usted calcular la profundidad de las restauraciones? Frecuencia Porcentaje

\begin{tabular}{lllr} 
Válidos & No & 19 & 47,5 \\
& Sí & 21 & 52,5 \\
& Total & 40 & 100,0 \\
\hline
\end{tabular}

El 52,5\% afirmó que sí podía calcular la profundidad de las restauraciones efectuadas a los pacientes que acudían a las consultas externas.

P6) ¿Sabe a qué distancia polimeriza el material fotocurado?

¿Sabe a qué distancia polimeriza el material fotocurado? Frecuencia Porcentaje

\begin{tabular}{lllr}
\hline Válidos & No & 17 & 42,5 \\
& Sí & 23 & 57,5 \\
& Total & 40 & 100,0 \\
\hline
\end{tabular}

Un mayoritario $57,5 \%$ posee conocimiento sobre la distancia a que se polimeriza el material fotocurado, mientras que un $42,5 \%$ señala desconocer la distancia.

P7) ¿Usted cree que los materiales de $3 \mathrm{~mm}$ de profundidad polimericen en su totalidad?

¿Usted cree que los materiales de $3 \mathrm{~mm}$ de profundidad polimericen en su totalidad?

Frecuencia Porcentaje

\begin{tabular}{lllr}
\hline Válidos & No & 14 & 35,0 \\
& Sí & 26 & 65,0 \\
& Total & 40 & 100,0 \\
\hline
\end{tabular}

Un 65\% de los encuestados señaló desconocer la acción de los materiales cuando se efectúa la polimerización. El 35\% respondió afirmativamente.

P8) Terminada la restauración por fotocurado profunda, ¿tiene la certeza de que dicho material ha sido polimerizado adecuadamente?

Terminada la restauración por fotocurado profunda, ¿tiene la certeza de que dicho material ha sido polimerizado adecuadamente?

Frecuencia Porcentaje

\begin{tabular}{rlrr}
\hline Válidos & No & 22 & 55,0 \\
& Sí & 18 & 45,0 \\
& Total & 40 & 100,0 \\
\hline
\end{tabular}

El 55,0\% tiene la certeza deque dicho material ha sido polimerizado adecuadamente en el momento de la restauración por fotocurado, y el 45,0 señaló no tener dicha certeza.

\section{Variable independiente: polimerización} Indicador: dureza de polimerización

\section{P9) ¿Conoce el concepto de polimerización?}

¿Conoce el concepto de polimerización?

\begin{tabular}{clcc}
\hline Válidos & & Frecuencia & Porcentaje \\
& No & 17 & 42,5 \\
& Sí & 23 & 57,5 \\
& Total & 40 & 100,0 \\
\hline
\end{tabular}


El 57,5\% conoce y maneja el concepto de polimerización, puesto que es una tecnología de reciente introducción y ya es enseñada en las prácticas preprofesionales y en el internado que efectúan los futuros odontólogos.

P10) ¿Los materiales polimerizables son mejores en restauraciones grandes y profundas?

¿Los materiales polimerizables son mejores en restauraciones grandes y profundas?

\section{Frecuencia Porcentaje}

\begin{tabular}{llrr}
\hline Válidos & No & 24 & 60,0 \\
& Sí & 16 & 40,0 \\
& Total & 40 & 100,0 \\
\hline
\end{tabular}

Un mayoritario $60,0 \%$ contestó afirmativamente en el sentido de que conocía la aplicación de los materiales polimerizables para mejorar los trabajos de restauraciones grandes y profundas.

\section{P11) ¿Usted cree que cuando el material es duro está completamente polimerizado? \\ ¿Usted cree que cuando el material es duro está completamente polimerizado?}

\section{Frecuencia Porcentaje}

\begin{tabular}{lllr}
\hline Válidos & No & 20 & 50,0 \\
& Sí & 20 & 50,0 \\
& Total & 40 & 100,0
\end{tabular}

Las respuestas están divididas en igual porcentaje respecto a esta pregunta, lo que evidencia que no se tiene una total seguridad sobre la dureza del material polimerizado.

\section{P12) ¿En cuántas horas el material restaurador polimerizado completa su polimerización? \\ ¿En cuántas horas el material restaurador polimerizado completa su polimerización?}

\section{Frecuencia Porcentaje}

\begin{tabular}{llrr}
\hline Válidos & No & 19 & 47,5 \\
& Sí & 21 & 52,5 \\
& Total & 40 & 100,0 \\
\hline
\end{tabular}

\section{Variable independiente: materiales dentales}

\section{Indicador: sensibilidad a la fuente de luz}

\section{P13) ¿Utiliza con frecuencia material estético fotopolimerizable en el sector anterior?}

¿Utiliza con frecuencia material estético fotopolimerizable en el sector anterior?

\section{Frecuencia Porcentaje}

\begin{tabular}{llrr}
\hline Válidos & No & 19 & 47,5 \\
& Sí & 21 & 52,5 \\
& Total & 40 & 100,0 \\
\hline
\end{tabular}

La respuesta fue afirmativamente contestada por el $52,5 \%$ del total de encuestados, mientras que el $47,5 \%$ contestó que no utiliza con frecuencia dicho material.

\section{P14) ¿Utiliza con frecuencia material restaurador fotopolimerizable en el sector posterior?}

¿Utiliza con frecuencia material restaurador fotopolimerizable en el sector posterior?

Frecuencia Porcentaje

\begin{tabular}{lllr}
\hline Válidos & No & 20 & 50,0 \\
& Sí & 20 & 50,0 \\
& Total & 40 & 100,0 \\
\hline
\end{tabular}

Respecto al uso del material restaurador fotopolimerizable en el sector posterior, las opiniones están divididas a favor $(50,0 \%)$ y en contra (50,0\%).

\section{P15) ¿Utiliza siempre base en cavidades profun- das?}

¿Utiliza siempre base en cavidades profundas?

Frecuencia Porcentaje

\begin{tabular}{lllr}
\hline Válidos & No & 17 & 42,5 \\
& Sí & 23 & 57,5 \\
& Total & 40 & 100,0 \\
\hline
\end{tabular}

El 57,5\% utiliza siempre base en cavidades profundas, mientras que el restante $42,5 \%$ no la utiliza. 
P16) ¿Tiene dificultad para llevar la fuente de luz hacia el material?

¿Tiene dificultad para llevar la fuente de luz hacia el material? Frecuencia Porcentaje

\begin{tabular}{lllr}
\hline Válidos & No & 17 & 42,5 \\
& Sí & 23 & 57,5 \\
& Total & 40 & 100,0 \\
\hline
\end{tabular}

El 57,5\% de los encuestados señaló que tiene dificultades para llevar la fuente de luz hacia el material cuando está aplicando esta técnica, y el $42,5 \%$ restante opinó que no tiene dificultades o problema alguno.

\section{P17) Utiliza otros aditamentos para disminuir la distancia de la punta de la fuente de luz al material fotocurado?} ¿Utiliza otros aditamentos para disminuir la distancia
de la punta de la fuente de luz al material fotocurado?

\section{Frecuencia Porcentaje}

\begin{tabular}{llrr}
\hline Válidos & No & 19 & 47,5 \\
& Sí & 21 & 52,5 \\
& Total & 40 & 100,0 \\
\hline
\end{tabular}

El 52,5\% de los encuestados respondió que utiliza otros aditamentos con la finalidad de disminuir la distancia de la punta de la fuente de luz al material fotocurado, mientras que el $47,5 \%$ señaló lo contrario.

Variable independiente: prototipo de medición de profundidad

\section{Indicador: diseño, estructura del prototipo}

P18) ¿Conoce usted un prototipo dental que sirve para medir la profundidad de polimerización?

¿Conoce usted un prototipo dental que sirve para medir profundidad de polimerización?

Frecuencia Porcentaje

\begin{tabular}{llrr}
\hline Válidos & No & 19 & 47,5 \\
& Sí & 21 & 52,5 \\
& Total & 40 & 100,0 \\
\hline
\end{tabular}

El 52,5\% señaló su conocimiento de este prototipo dental cuya utilidad es destinada para medir la profundidad de polimerización, mientras que el 52,5\% manifestó su desconocimiento.

\section{P19) Si existiera, ¿usted cree que ayudaría para mejorar su labor?}

Si existiera, ¿usted cree que ayudaria para mejorar su labor?

Frecuencia Porcentaje

\begin{tabular}{rlrr}
\hline Válidos & No & 17 & 42,5 \\
& Sí & 23 & 57,5 \\
& Total & 40 & 100,0 \\
\hline
\end{tabular}

P20) ¿El prototipo que sirva para medir profundidad de restauraciones fotopolimerizables ayudaria con la ciencia de los materiales fotopolimerizables? ¿El prototipo que sirva para medir profundidad de
restauraciones fotopolimerizables ayudaría con
la ciencia de los materiales fotopolimerizables?

\begin{tabular}{llcc} 
& & Frecuencia & Porcentaje \\
\hline Válidos & No & 14 & 35,0 \\
& Sí & 26 & 65,0 \\
& Total & 40 & 100,0 \\
\hline
\end{tabular}

Un mayoritario $65,0 \%$ consideró que el uso de este prototipo sirve para medir la profundidad de restauraciones fotopolimerizables, y en sentido contrario opinó el 35,0\% restante.

\section{P21) ¿Usted cree que es costoso diseñar un prototi- po para este propósito? \\ ¿Usted cree que es costoso diseñar un prototipo para este propósito?}

\begin{tabular}{clcc} 
& & Frecuencia & Porcentaje \\
\hline Válidos & No & 22 & 55,0 \\
& Sí & 18 & 45,0 \\
& Total & 40 & 100,0 \\
\hline
\end{tabular}

El 55,0\% señaló que no es muy costoso diseñar un prototipo para este propósito, y el 45 $\%$ consideró que sí era costoso diseñar tal prototipo. 


\section{DISCUSIÓN}

\section{Contrastación de hipótesis}

\section{Hipótesis principal}

Ho: Con el prototipo para medir la profundidad de fotopolimerización no se mejorará la fotopolimerización de restauraciones dentales.

H1: Con el prototipo para medir la profundidad de fotopolimerización se mejorará la fotopolimerización de restauraciones dentales.

Tabla de contingencia. ¿Puede usted calcular la profundidad de las restauraciones? ¿Tiene conocimiento a qué distancia polimerizan los materiales fotocurados?

\begin{tabular}{llccc} 
& & \multicolumn{2}{c}{$\begin{array}{c}\text { ¿Tiene conocimiento a } \\
\text { qué distancia polimerizan lo } \\
\text { materiales fotocurados? }\end{array}$} & Total \\
\% del total & No & Sí & \\
\hline ¿Puede usted calcular & No & $32,5 \%$ & $15,0 \%$ & $47,5 \%$ \\
la profundidad de las & Sí & $10,0 \%$ & $42,5 \%$ & $52,5 \%$ \\
Restauraciones? & & & & \\
Total & & $42,5 \%$ & $57,5 \%$ & $100,0 \%$ \\
\hline
\end{tabular}

Pruebas de Chi-cuadrado

\begin{tabular}{|c|c|c|c|c|c|}
\hline & Valor & $\mathrm{gl}$ & $\begin{array}{l}\text { Sig. asintótica } \\
\text { (bilateral) }\end{array}$ & $\begin{array}{l}\text { Sig. exacta } \\
\text { (bilateral) }\end{array}$ & $\begin{array}{l}\text { Sig. exacta } \\
\text { (unilateral) }\end{array}$ \\
\hline Chi-cuadrado de Pearson & $9,950^{\mathrm{b}}$ & 1 & 0,002 & & \\
\hline Corrección por continuidad $^{a}$ & 8,033 & 1 & 0,005 & & \\
\hline Razón de verosimilitud & 10,399 & 1 & 0,001 & & \\
\hline Estadístico exacto de Fisher & & & & 0,003 & 0,002 \\
\hline Asociación lineal por lineal & 9,702 & 1 & 0,002 & & \\
\hline $\mathrm{N}^{0}$ de casos válidos & 40 & & & & \\
\hline
\end{tabular}

Medidas simétricas

\begin{tabular}{|c|c|c|c|c|c|}
\hline & & Valor & $\begin{array}{l}\text { Error tip. } \\
\text { asint. }\end{array}$ & T. aproximada ${ }^{b}$ & $\begin{array}{c}\text { Sig. } \\
\text { aproximada }\end{array}$ \\
\hline Intervalo por intervalo & R de Pearson & 0,499 & 0,137 & 3,547 & $0,001^{\mathrm{c}}$ \\
\hline Ordinal por ordinal & Correlación de Spearman & 0,499 & 0,137 & 3,547 & $0,001^{\mathrm{c}}$ \\
\hline $\mathrm{N}^{0}$ de casos válidos & & 40 & & & \\
\hline
\end{tabular}

a. Asumiendo la hipótesis alternativa.

b. Empleando el error típico asintótico basado en la hipótesis nula.

c. Basada en la aproximación normal. 
¿Tiene conocimiento a qué distancia polimerizan los materiales fotocurados? ¿Usted cree que los materiales de $3 \mathrm{~mm}$ de profundidad polimericen en su totalidad?

Tabla de contingencia

\begin{tabular}{|c|c|c|c|c|}
\hline \multirow[b]{2}{*}{$\%$ del total } & & \multicolumn{2}{|c|}{$\begin{array}{l}\text { ¿Usted cree que los materiales } \\
\text { de } 3 \mathrm{~mm} \text { de profundidad } \\
\text { polimericen en su totalidad? }\end{array}$} & \multirow[t]{2}{*}{ Total } \\
\hline & & No & Sí & \\
\hline $\begin{array}{l}\text { ¿Tiene conocimiento a qué } \\
\text { distancia polimerizan los } \\
\text { materiales fotocurados? }\end{array}$ & $\begin{array}{l}\text { No } \\
\text { Sí }\end{array}$ & $\begin{array}{l}25,0 \% \\
10,0 \%\end{array}$ & $\begin{array}{l}17,5 \% \\
47,5 \%\end{array}$ & $\begin{array}{l}42,5 \% \\
57,5 \%\end{array}$ \\
\hline Total & & $35,0 \%$ & $65,0 \%$ & $100,0 \%$ \\
\hline
\end{tabular}

Pruebas de Chi-cuadrado

\begin{tabular}{|c|c|c|c|c|c|}
\hline & Valor & $\mathrm{gl}$ & $\begin{array}{l}\text { Sig. asintótica } \\
\text { (bilateral) }\end{array}$ & $\begin{array}{l}\text { Sig. exacta } \\
\text { (bilateral) }\end{array}$ & $\begin{array}{l}\text { Sig. exacta } \\
\text { (unilateral) }\end{array}$ \\
\hline Chi-cuadrado de Pearson & $7,376^{\mathrm{b}}$ & 1 & 0,007 & & \\
\hline Corrección por continuidad ${ }^{\mathrm{a}}$ & 5,667 & 1 & 0,017 & & \\
\hline Razón de verosimilitud & 7,507 & 1 & 0,006 & & \\
\hline Estadístico exacto de Fisher & & & & 0,009 & 0,008 \\
\hline Asociación lineal por lineal & 7,191 & 1 & 0,007 & & \\
\hline $\mathrm{N}^{0}$ de casos válidos & 40 & & & & \\
\hline
\end{tabular}

a. Calculado sólo para una tabla de $2 \times 2$.

b. 0 casillas $(0 \%)$ tienen una frecuencia esperada inferior a 5. La frecuencia mínima esperada es 5,95.

\begin{tabular}{|c|c|c|c|c|c|}
\hline \multicolumn{6}{|c|}{ Medidas simétricas } \\
\hline & & Valor & $\begin{array}{c}\text { Error tip. } \\
\text { asint. }\end{array}$ & T. aproximada ${ }^{\mathrm{b}}$ & $\begin{array}{c}\text { Sig. } \\
\text { aproximada }\end{array}$ \\
\hline Intervalo por intervalo & R de Pearson & 0,429 & 0,145 & 2,931 & $0,006^{c}$ \\
\hline Ordinal por ordinal & Correlación de Spearman & 0,429 & 0,145 & 2,931 & $0,006^{c}$ \\
\hline $\mathrm{N}^{0}$ de casos válidos & & 40 & & & \\
\hline
\end{tabular}

¿Puede usted calcular la profundidad de las restauraciones? Terminada la restauración por fotocurado profunda, ¿ tiene la certeza de que dicho material ha sido polimerizado adecuadamente?

Tabla de contingencia

\begin{tabular}{|c|c|c|c|c|}
\hline \multirow[b]{2}{*}{$\%$ del total } & & $\begin{array}{r}\text { Terminad } \\
\text { por fotocu } \\
\text { ¿tiene la } \\
\text { dicho m } \\
\text { polimerizad }\end{array}$ & $\begin{array}{l}\text { luración } \\
\text { rofunda, } \\
\text { de que } \\
\text { ha sido } \\
\text { ladamente? }\end{array}$ & \multirow[t]{2}{*}{ Total } \\
\hline & & No & Sí & \\
\hline ¿Puede usted calcular & No & $35,0 \%$ & $12,5 \%$ & $47,5 \%$ \\
\hline $\begin{array}{l}\text { la profundidad de las } \\
\text { restauraciones? }\end{array}$ & Sí & $20,0 \%$ & $32,5 \%$ & $52,5 \%$ \\
\hline Total & & $55,0 \%$ & $45,0 \%$ & $100,0 \%$ \\
\hline
\end{tabular}


Pruebas de Chi-cuadrado

\begin{tabular}{|c|c|c|c|c|c|}
\hline & Valor & $\mathrm{gl}$ & $\begin{array}{l}\text { Sig. asintótica } \\
\text { (bilateral) }\end{array}$ & $\begin{array}{l}\text { Sig. exacta } \\
\text { (bilateral) }\end{array}$ & $\begin{array}{l}\text { Sig. exacta } \\
\text { (unilateral) }\end{array}$ \\
\hline Chi-cuadrado de Pearson & $5,105^{b}$ & 1 & 0,024 & & \\
\hline Corrección por continuidad ${ }^{a}$ & 3,768 & 1 & 0,052 & & \\
\hline Razón de verosimilitud & 5,240 & 1 & 0,022 & & \\
\hline Estadístico exacto de Fisher & & & & 0,031 & 0,025 \\
\hline Asociación lineal por lineal & 4,977 & 1 & 0,026 & & \\
\hline $\mathrm{N}^{0}$ de casos válidos & 40 & & & & \\
\hline
\end{tabular}

Medidas simétricas

\begin{tabular}{llcccc} 
& & Valor & $\begin{array}{c}\text { Error tip. } \\
\text { asint. }^{{ }^{2}}\end{array}$ & $\begin{array}{c}\text { T. aproximada } \\
\text { b }\end{array}$ & $\begin{array}{c}\text { Sig. } \\
\text { aproximada }\end{array}$ \\
\hline $\begin{array}{l}\text { Intervalo por intervalo } \\
\text { Ordinal por ordinal }\end{array}$ & R de Pearson & 0,357 & 0,147 & 2,358 & $0,024^{c}$ \\
$\mathrm{~N}^{0}$ de casos válidos & & 40 & & 2,358 & $0,024^{c}$ \\
\hline
\end{tabular}

a. Asumiendo la hipótesis alternativa.

b. Empleando el error típico asintótico basado en la hipótesis nula.

c. Basada en la aproximación normal.

¿Tiene conocimiento a qué distancia polimerizan los materiales fotocurados? Terminada la restauración por fotocurado profunda, ¿tiene la certeza de que dicho material ha sido polimerizado adecuadamente?

Tabla de contingencia

\begin{tabular}{|c|c|c|c|c|}
\hline \multirow[b]{2}{*}{$\%$ del total } & & $\begin{array}{l}\text { Terminad } \\
\text { por fotoc } \\
\text { ¿tiene la } \\
\text { dicho } n \\
\text { polimerizad }\end{array}$ & $\begin{array}{l}\text { rouración } \\
\text { rofunda, } \\
\text { de que } \\
\text { ha sido } \\
\text { ladamente? }\end{array}$ & \multirow[t]{2}{*}{ Total } \\
\hline & & No & Sí & \\
\hline $\begin{array}{l}\text { ¿Tiene conocimiento a qué } \\
\text { distancia polimerizan los } \\
\text { materiales fotocurados? }\end{array}$ & $\begin{array}{l}\text { No } \\
\text { Sí }\end{array}$ & $\begin{array}{l}35,0 \% \\
20,0 \%\end{array}$ & $\begin{array}{r}7,5 \% \\
37,5 \%\end{array}$ & $\begin{array}{l}42,5 \% \\
57,5 \%\end{array}$ \\
\hline Total & & $55,0 \%$ & $45,0 \%$ & $100,0 \%$ \\
\hline
\end{tabular}

Pruebas de Chi-cuadrado

\begin{tabular}{|c|c|c|c|c|c|}
\hline & Valor & $\mathrm{gl}$ & $\begin{array}{l}\text { Sig. asintótica } \\
\text { (bilateral) }\end{array}$ & $\begin{array}{l}\text { Sig. exacta } \\
\text { (bilateral) }\end{array}$ & $\begin{array}{l}\text { Sig. exacta } \\
\text { (unilateral) }\end{array}$ \\
\hline Chi-cuadrado de Pearson & $8,937^{\mathrm{b}}$ & & 0,003 & & \\
\hline Corrección por continuidad ${ }^{a}$ & 7,119 & & 0,008 & & \\
\hline Razón de verosimilitud & 9,487 & & 0,002 & & \\
\hline Estadístico exacto de Fisher & & & & 0,004 & 0,003 \\
\hline Asociación lineal por lineal & 8,714 & & 0,003 & & \\
\hline $\mathrm{N}^{0}$ de casos válidos & 40 & & & & \\
\hline
\end{tabular}

a. Calculado sólo para una tabla de $2 \times 2$.

b. 0 casillas $(0 \%)$ tienen una frecuencia esperada inferior a 5 . La frecuencia mínima esperada es 7,65. 


\begin{tabular}{llcccc} 
& \multicolumn{2}{c}{ Medidas simétricas } & & \\
& & Valor & $\begin{array}{c}\text { Error tip. } \\
\text { asint. }^{2}\end{array}$ & $\begin{array}{c}\text { T. aproximada } \\
\text { b }\end{array}$ & $\begin{array}{c}\text { Sig. } \\
\text { aproximada }\end{array}$ \\
\hline Intervalo por intervalo & R de Pearson & 0,473 & 0,134 & 3,307 & $0,002^{\mathrm{c}}$ \\
Ordinal por ordinal & Correlación de Spearman & 0,473 & 0,134 & 3,307 & $0,002^{\mathrm{c}}$ \\
$\mathrm{N}^{0}$ de casos válidos & & 40 & & & \\
\hline
\end{tabular}

a. Asumiendo la hipótesis alternativa.

b. Empleando el error típico asintótico basado en la hipótesis nula.

c. Basada en la aproximación normal.

¿Usted cree que los materiales de $3 \mathrm{~mm}$ de profundidad polimericen en su totalidad? Terminada la restauración por fotocurado profunda, ¿tiene la certeza de que dicho material ha sido polimerizado adecuadamente?

\section{Tabla de contingencia}

\begin{tabular}{lcccc} 
& & \multicolumn{2}{c}{$\begin{array}{c}\text { Terminada la restauración } \\
\text { por fotocurado profunda, } \\
\text { ¿'tiene la certeza de que } \\
\text { dicho material ha sido } \\
\text { polimerizado adecuadamente? }\end{array}$} & Total \\
$\begin{array}{l}\text { \% del total } \\
\text { ¿Usted cree que los }\end{array}$ & No & $32,5 \%$ & $2,5 \%$ & $35,0 \%$ \\
$\begin{array}{l}\text { materiales de 3mm de } \\
\text { profundidad polimericen } \\
\text { en su totalidad? }\end{array}$ & Sí & $22,5 \%$ & $42,5 \%$ & $65,0 \%$ \\
$\begin{array}{l}\text { Total } \\
\text { Total }\end{array}$ & & $55,0 \%$ & $45,0 \%$ & $100,0 \%$ \\
\hline
\end{tabular}

Pruebas de Chi-cuadrado

\begin{tabular}{|c|c|c|c|c|c|}
\hline & Valor & $\mathrm{gl}$ & $\begin{array}{l}\text { Sig. asintótica } \\
\text { (bilateral) }\end{array}$ & $\begin{array}{l}\text { Sig. exacta } \\
\text { (bilateral) }\end{array}$ & $\begin{array}{l}\text { Sig. exacta } \\
\text { (unilateral) }\end{array}$ \\
\hline Chi-cuadrado de Pearson & $12,472^{b}$ & & 0,000 & & \\
\hline Corrección por continuidad ${ }^{a}$ & 10,230 & & 0,001 & & \\
\hline Razón de verosimilitud & 14,304 & & 0,000 & & \\
\hline Estadístico exacto de Fisher & & & & 0,001 & 0,000 \\
\hline Asociación lineal por lineal & 12,160 & & 0,000 & & \\
\hline $\mathrm{N}^{0}$ de casos válidos & 40 & & & & \\
\hline
\end{tabular}

a. Calculado sólo para una tabla de $2 \times 2$.

b. 0 casillas (0\%) tienen una frecuencia esperada inferior a 5. La frecuencia mínima esperada es 6,30.

Medidas simétricas

\begin{tabular}{|c|c|c|c|c|c|}
\hline & & Valor & $\begin{array}{l}\text { Error tip. } \\
\text { asint. }^{\text {a }}\end{array}$ & T. aproximada ${ }^{b}$ & $\begin{array}{c}\text { Sig. } \\
\text { aproximada }\end{array}$ \\
\hline Intervalo por intervalo & R de Pearson & 0,558 & 0,112 & 4,149 & $0,000^{c}$ \\
\hline Ordinal por ordinal & Correlación de Spearman & 0,558 & 0,112 & 4,149 & $0,000^{c}$ \\
\hline $\mathrm{N}^{0}$ de casos válidos & & 40 & & & \\
\hline
\end{tabular}

a. Asumiendo la hipótesis alternativa.

b. Empleando el error típico asintótico basado en la hipótesis nula.

c. Basada en la aproximación normal. 
¿Utiliza con frecuencia material restaurador fotopolimerizable en el sector posterior? ¿El prototipo que sirva para medir profundidad de restauraciones fotopolimerizables ayudaría con la ciencia de los materiales fotopolimerizables?

\section{Tabla de contingencia}

\begin{tabular}{|c|c|c|c|c|}
\hline \multirow[b]{2}{*}{$\%$ del total } & & \multicolumn{2}{|c|}{$\begin{array}{c}\text { ¿El prototipo que sirva } \\
\text { para medir profundidad } \\
\text { de restauraciones } \\
\text { fotopolimerizables ayudaría } \\
\text { con la ciencia de los materiales } \\
\text { fotopolimerizables? }\end{array}$} & \multirow[t]{2}{*}{ Total } \\
\hline & & No & Sí & \\
\hline $\begin{array}{l}\text { ¿Utiliza con frecuencia } \\
\text { material restaurador }\end{array}$ & No & $25,0 \%$ & $25,0 \%$ & $50,0 \%$ \\
\hline $\begin{array}{l}\text { fotopolimerizable en } \\
\text { el sector posterior? }\end{array}$ & Sí & $10,0 \%$ & $40,0 \%$ & $50,0 \%$ \\
\hline Total & & $35,0 \%$ & $65,0 \%$ & $100,0 \%$ \\
\hline
\end{tabular}

Pruebas de Chi-cuadrado

\begin{tabular}{lccccc} 
& Valor & gl & $\begin{array}{c}\text { Sig. asintótica } \\
\text { (bilateral) }\end{array}$ & $\begin{array}{c}\text { Sig. exacta } \\
\text { (bilateral) }\end{array}$ & $\begin{array}{c}\text { Sig. exacta } \\
\text { (unilateral) }\end{array}$ \\
\hline Chi-cuadrado de Pearson & $3,956^{\mathrm{b}}$ & 1 & 0,047 & & \\
Corrección por continuidad ${ }^{\mathrm{a}}$ & 2,747 & 1 & 0,097 & & \\
\hline Razón de verosimilitud & 4,054 & 1 & 0,044 & & 0,096 \\
\hline Estadístico exacto de Fisher & & & & & \\
\hline Asociación lineal por lineal & 3,857 & 1 & 0,050 & & \\
$\mathrm{~N}^{0}$ de casos válidos & 40 & & & & \\
\hline
\end{tabular}

a. Calculado sólo para una tabla de $2 \times 2$.

b. 0 casillas $(0 \%)$ tienen una frecuencia esperada inferior a 5 . La frecuencia mínima esperada es 7,00.

\begin{tabular}{llcccc} 
& \multicolumn{2}{c}{ Medidas simétricas } & & \\
& & Valor & $\begin{array}{c}\text { Error tip. } \\
\text { asint. }^{{ }^{*}}\end{array}$ & $\begin{array}{c}\text { T. aproximada } \\
\text { b }\end{array}$ & $\begin{array}{c}\text { Sig. } \\
\text { aproximada }\end{array}$ \\
\hline Intervalo por intervalo & R de Pearson & 0,314 & 0,148 & 2,042 & $0,048^{c}$ \\
Ordinal por ordinal & Correlación de Spearman & 0,314 & 0,148 & 2,042 & $0,048^{c}$ \\
$\mathrm{~N}^{0}$ de casos válidos & & 40 & & & \\
\hline
\end{tabular}

a. Asumiendo la hipótesis alternativa.

b. Empleando el error típico asintótico basado en la hipótesis nula.

c. Basada en la aproximación normal. 
¿Utiliza con frecuencia material restaurador fotopolimerizable en el sector posterior? ¿Conoce usted un prototipo dental que sirve para medir profundidad de polimerización?

Tabla de contingencia

\begin{tabular}{|c|c|c|c|c|}
\hline \multirow[b]{2}{*}{$\%$ del total } & & \multicolumn{2}{|c|}{$\begin{array}{l}\text { ¿Conoce usted un } \\
\text { prototipo dental que sirve } \\
\text { para medir profundidad } \\
\text { de polimerizacón? }\end{array}$} & \multirow[t]{2}{*}{ Total } \\
\hline & & No & Sí & \\
\hline $\begin{array}{l}U \text { tiliza con frecuencia } \\
\text { material restaurador }\end{array}$ & No & $37,5 \%$ & $12,5 \%$ & $50,0 \%$ \\
\hline $\begin{array}{l}\text { fotopolimerizable en } \\
\text { el sector posterior? }\end{array}$ & Sí & $10,0 \%$ & $40,0 \%$ & $50,0 \%$ \\
\hline Total & & $47,5 \%$ & $52,5 \%$ & $100,0 \%$ \\
\hline
\end{tabular}

Pruebas de Chi-cuadrado

\begin{tabular}{|c|c|c|c|c|c|}
\hline & Valor & $\mathrm{gl}$ & $\begin{array}{l}\text { Sig. asintótica } \\
\text { (bilateral) }\end{array}$ & $\begin{array}{l}\text { Sig. exacta } \\
\text { (bilateral) }\end{array}$ & $\begin{array}{l}\text { Sig. exacta } \\
\text { (unilateral) }\end{array}$ \\
\hline Chi-cuadrado de Pearson & $12,130^{\mathrm{b}}$ & 1 & 0,000 & & \\
\hline Corrección por continuidad ${ }^{a}$ & 10,025 & 1 & 0,002 & & \\
\hline Razón de verosimilitud & 12,842 & 1 & 0,000 & & \\
\hline Estadístico exacto de Fisher & & & & 0,001 & 0,001 \\
\hline Asociación lineal por lineal & 11,827 & 1 & 0,001 & & \\
\hline $\mathrm{N}^{0}$ de casos válidos & 40 & & & & \\
\hline
\end{tabular}

a. Calculado sólo para una tabla de $2 \times 2$.

b. 0 casillas $(0 \%)$ tienen una frecuencia esperada inferior a 5. La frecuencia mínima esperada es 9,50.

\begin{tabular}{|c|c|c|c|c|c|}
\hline \multicolumn{6}{|c|}{ Medidas simétricas } \\
\hline & & Valor & $\begin{array}{c}\text { Error tip. } \\
\text { asint. }^{a}\end{array}$ & T. aproximada ${ }^{\mathrm{b}}$ & $\begin{array}{c}\text { Sig. } \\
\text { aproximada }\end{array}$ \\
\hline Intervalo por intervalo & R de Pearson & 0,551 & 0,132 & 4,067 & $0,000^{c}$ \\
\hline Ordinal por ordinal & Correlación de Spearman & 0,551 & 0,132 & 4,067 & $0,000^{c}$ \\
\hline $\mathrm{N}^{0}$ de casos válidos & & 40 & & & \\
\hline
\end{tabular}

a. Asumiendo la hipótesis alternativa.

b. Empleando el error típico asintótico basado en la hipótesis nula.

c. Basada en la aproximación normal. 
Tabla de contingencia. ¿El prototipo que sirva para medir profundidad de restauraciones fotopolimerizable ayudaría con la ciencia de los materiales fotopolimerizables? ¿Usted cree que es costoso diseñar un prototipo para este propósito?

\begin{tabular}{|c|c|c|c|c|}
\hline \multirow[b]{2}{*}{$\%$ del total } & & \multicolumn{2}{|c|}{$\begin{array}{c}\text { ¿Usted cree que es } \\
\text { costoso diseñar un prototipo } \\
\text { para este propósito? }\end{array}$} & \multirow{2}{*}{ Total } \\
\hline & & No & Sí & \\
\hline $\begin{array}{l}\text { ¿El prototipo que sirva } \\
\text { para medir profundidad }\end{array}$ & No & $32,5 \%$ & $2,5 \%$ & $35,0 \%$ \\
\hline $\begin{array}{l}\text { de restauraciones } \\
\text { fotopolimerizable } \\
\text { ayudaría con la ciencia } \\
\text { de los materiales } \\
\text { fotopolimerizables? }\end{array}$ & Sí & $22,5 \%$ & $42,5 \%$ & $65,0 \%$ \\
\hline Total & & $55,0 \%$ & $45,0 \%$ & $100,0 \%$ \\
\hline
\end{tabular}

Pruebas de Chi-cuadrado

\begin{tabular}{lccccc} 
& Valor & gl & $\begin{array}{c}\text { Sig. asintótica } \\
\text { (bilateral) }\end{array}$ & $\begin{array}{c}\text { Sig. exacta } \\
\text { (bilateral) }\end{array}$ & $\begin{array}{c}\text { Sig. exacta } \\
\text { (unilateral) }\end{array}$ \\
\hline Chi-cuadrado de Pearson & $12,472^{\mathrm{b}}$ & 1 & 0,000 & & \\
\hline Corrección por continuidad & 10,230 & 1 & 0,001 & & \\
Razón de verosimilitud & 14,304 & 1 & 0,000 & & 0,000 \\
\hline Estadístico exacto de Fisher & & & & & \\
\hline Asociación lineal por lineal & 12,160 & 1 & 0,000 & & \\
\hline No de casos válidos & 40 & & & & \\
\hline
\end{tabular}

a. Calculado sólo para una tabla de $2 \times 2$.

b. 0 casillas $(0 \%)$ tienen una frecuencia esperada inferior a 5 . La frecuencia mínima esperada es 6,30.

Medidas simétricas

\begin{tabular}{llcccc} 
& & Valor & $\begin{array}{c}\text { Error tip. } \\
\text { asint. }^{{ }^{2}}\end{array}$ & $\begin{array}{c}\text { T. aproximada } \\
\text { intervalo por intervalo }\end{array}$ & $\begin{array}{c}\text { Sig. } \\
\text { aproximada }\end{array}$ \\
\hline Ordinal por ordinal & Correlación de Spearman & 0,558 & 0,112 & 4,149 & 0,000 \\
$\mathrm{~N}^{0}$ de casos válidos & & 40 & & 4,149 & 0,000 \\
\hline
\end{tabular}

a. Asumiendo la hipótesis alternativa.

b. Empleando el error típico asintótico basado en la hipótesis nula.

c. Basada en la aproximación normal. 
Si existiera, ¿usted cree que ayudaría para mejorar su labor? ¿El prototipo que sirva para medir profundidad de restauraciones fotopolimerizables ayudaría con la ciencia de los materiales fotopolimerizables?

Tabla de contingencia

\begin{tabular}{|c|c|c|c|c|}
\hline \multirow[b]{2}{*}{$\%$ del total } & & \multicolumn{2}{|c|}{$\begin{array}{c}\text { ¿El prototipo que sirva } \\
\text { para medir profundidad } \\
\text { de restauraciones } \\
\text { fotopolimerizables ayudaría } \\
\text { con la ciencia de los materiales } \\
\text { fotopolimerizables? }\end{array}$} & \multirow[t]{2}{*}{ Total } \\
\hline & & No & Sí & \\
\hline $\begin{array}{l}\text { Si existiera, ¿usted cree } \\
\text { que ayudaría para }\end{array}$ & No & $25,0 \%$ & $17,5 \%$ & $42,5 \%$ \\
\hline mejorar su labor? & Sí & $10,0 \%$ & $47,5 \%$ & $57,5 \%$ \\
\hline Total & & $35,0 \%$ & $65,0 \%$ & $100,0 \%$ \\
\hline
\end{tabular}

Pruebas de Chi-cuadrado

\begin{tabular}{|c|c|c|c|c|c|}
\hline & Valor & $\mathrm{gl}$ & $\begin{array}{l}\text { Sig. asintótica } \\
\text { (bilateral) }\end{array}$ & $\begin{array}{l}\text { Sig. exacta } \\
\text { (bilateral) }\end{array}$ & $\begin{array}{l}\text { Sig. exacta } \\
\text { (unilateral) }\end{array}$ \\
\hline Chi-cuadrado de Pearson & $7,376^{\mathrm{b}}$ & 1 & 0,007 & & \\
\hline Corrección por continuidad ${ }^{a}$ & 5,667 & 1 & 0,017 & & \\
\hline Razón de verosimilitud & 7,507 & 1 & 0,006 & & \\
\hline Estadístico exacto de Fisher & & & & 0,009 & 0,008 \\
\hline Asociación lineal por lineal & 7,191 & 1 & 0,007 & & \\
\hline No de casos válidos & 40 & & & & \\
\hline
\end{tabular}

a. Calculado sólo para una tabla de $2 \times 2$.

b. 0 casillas $(0 \%)$ tienen una frecuencia esperada inferior a 5. La frecuencia mínima esperada es 5,95.

Medidas simétricas

\begin{tabular}{|c|c|c|c|c|c|}
\hline & & Valor & $\begin{array}{c}\text { Error tip. } \\
\text { asint. }^{a}\end{array}$ & T. aproximada ${ }^{b}$ & $\begin{array}{l}\text { Sig. } \\
\text { aproximada }\end{array}$ \\
\hline Intervalo por intervalo & R de Pearson & 0,429 & 0,145 & 2,931 & $0,006^{c}$ \\
\hline Ordinal por ordinal & Correlación de Spearman & 0,429 & 0,145 & 2,931 & $0,006^{c}$ \\
\hline $\mathrm{N}^{0}$ de casos válidos & & 40 & & & \\
\hline
\end{tabular}

a. Asumiendo la hipótesis alternativa.

b. Empleando el error típico asintótico basado en la hipótesis nula.

c. Basada en la aproximación normal. 


\section{Si existiera, ¿usted cree que ayudaría para mejorar su labor? ¿Usted cree que es costoso diseñar un prototipo para este propósito?}

Tabla de contingencia

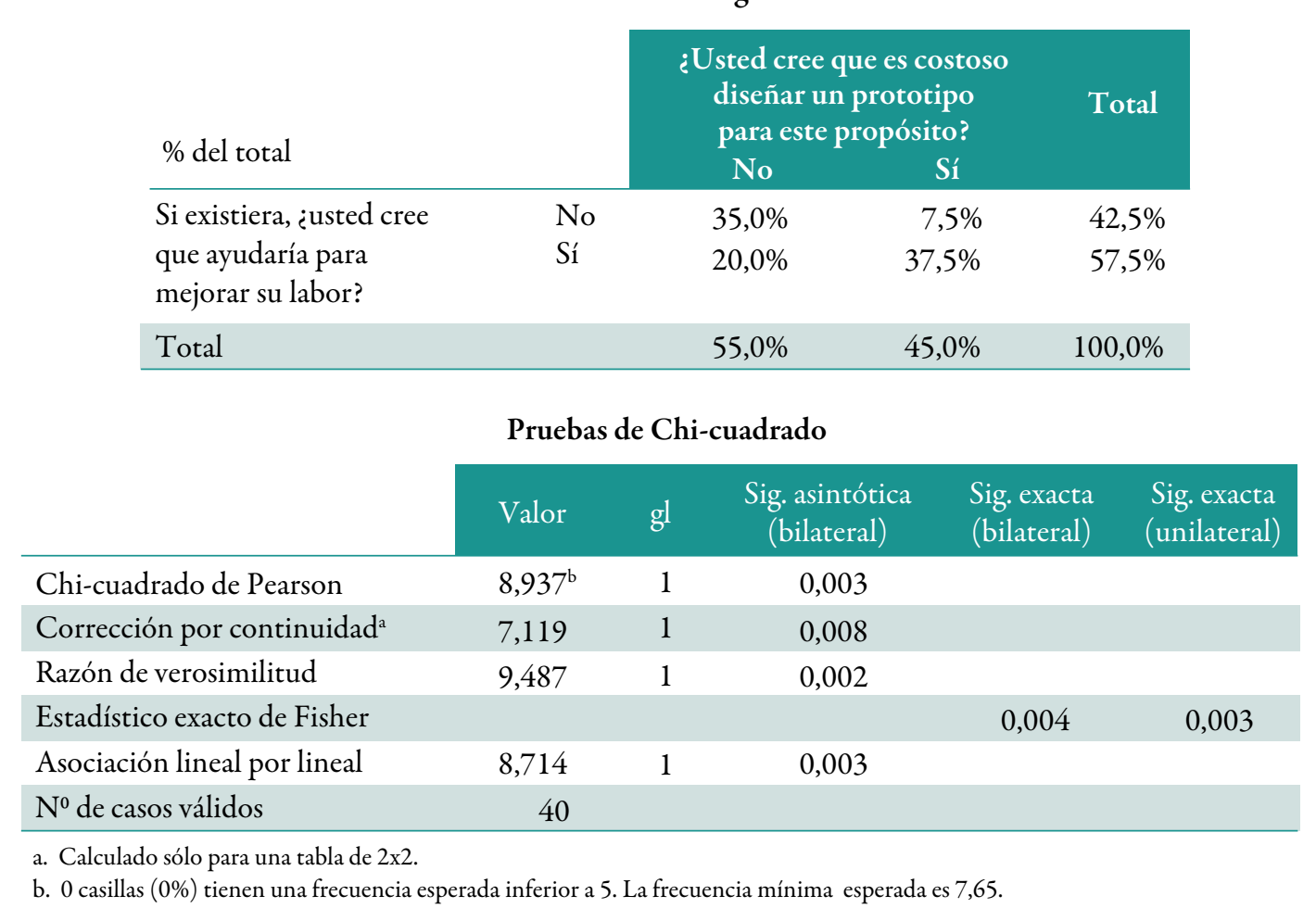

Medidas simétricas

\begin{tabular}{|c|c|c|c|c|c|}
\hline & & Valor & $\begin{array}{c}\text { Error tip. } \\
\text { asint. }^{\mathrm{a}}\end{array}$ & T. aproximada ${ }^{b}$ & $\begin{array}{c}\text { Sig. } \\
\text { aproximada }\end{array}$ \\
\hline Intervalo por intervalo & R de Pearson & 0,473 & 0,134 & 3,307 & $0,002^{\mathrm{c}}$ \\
\hline Ordinal por ordinal & Correlación de Spearman & 0,473 & 0,134 & 3,307 & $0,002^{\mathrm{c}}$ \\
\hline $\mathrm{N}^{0}$ de casos válidos & & 40 & & & \\
\hline
\end{tabular}

a. Asumiendo la hipótesis alternativa.

b. Empleando el error típico asintótico basado en la hipótesis nula.

c. Basada en la aproximación normal.

El p-value es menor a un $\alpha=0,05$, por lo que se rechaza la hipótesis planteada.

Existe suficiente evidencia estadística para afirmar que con el prototipo para medir la profundidad de fotopolimerización se mejorarán las restauraciones dentales, con un nivel de significación de 0,05 .

\section{RESULTADOS}

De la aplicación de las diversas técnicas de recopilación de información, podemos establecer los siguientes resultados:

1. El concepto de polimerización y su aplicación en la cirugía dental es una tecnología de reciente introducción y se viene experimentando desde las prácticas preprofesionales o internado que efectúan los futuros odontó- 
logos; por tanto, se tiene un dominio relativo de la aplicación de los materiales polimerizables cuando se trata de mejorar los trabajos de restauraciones grandes y profundas de los pacientes que acuden al servicio de Odontología del Hospital de la Policía Nacional del Perú.

2. Se presentan problemas secundarios con la aplicación de los materiales empleados, especialmente con la dureza que adquieren cuando están completamente polimerizados, pues estos varían en cuanto al tiempo de duración. De ahí que se hace necesario tener en consideración algunos factores destacados, como son:

a. La sensibilidad a la fuente de luz -necesaria para utilizar frecuentemente material estético fotopolimerizable en el sector anterior y posterior-, su uso recomendable aplicado en la base de cavidades profundas, junto a otros aditamentos para disminuir la distancia de la puesta de la fuente de luz al material fotocurado.

b. El diseño y estructura del prototipo dental que sirve para medir la profundidad de polimerización, el cual es considerado por un buen número de odontólogos como una ayuda para mejorar su labor, pues con él se podría medir la profundidad de las restauraciones fotopolimerizables, aplicando la ciencia de los materiales. Insistiendo en el diseño, su costo no es tan alto y puede ser solventado por los odontólogos.

3. Respecto a la profundidad de polimerización, la mayoría de profesionales señala que sí es posible calcular la profundidad de las restauraciones, porque se conoce también la distancia a que se polimeriza el material fotocurado (3 mm). Por lo tanto, terminada la restauración fotocurada profunda se podría aseverar que en gran parte dicho material ha sido polimerizado adecuadamente.
En síntesis, ha quedado comprobada la hipótesis planteada de acuerdo a los resultados obtenidos.

\section{CONCLUSIONES}

\section{Conclusiones parciales}

1. Entre los factores que influyen en la fotopolimerización de las restauraciones de las piezas dentarias destacan la contracción y el grado de conversión.

2. Entre los materiales de restauración que permiten mejorar la fotopolimerización en las piezas dentarias están las resinas compuestas.

3. Ha quedado demostrado que el diseño del prototipo elaborado con las características específicas contribuye a mejorar la fotopolimerización en las restauraciones de las piezas dentarias.

4. Los efectos de la fotopolimerización influyen en la profundidad de las restauraciones en las piezas dentarias.

\section{Conclusión general}

Existe suficiente evidencia estadística para afirmar que con el prototipo para medir la profundidad de fotopolimerización se mejorarán las restauraciones dentales de los pacientes que acuden a los consultorios de Odontología del Hospital Central Luis Sáenz de la Policía Nacional del Perú, como lo aseveran los odontólogos que laboran en dicha institución.

\section{RECOMENDACIONES}

1. El Hospital de la Policía Nacional del Perú, dentro de su Plan de Modernización de Tecnología Clínica, debiera mejorar o reempla- 
zar los equipos e instrumentos con los cuales atiende a los pacientes en los consultorios odontológicos. Los actuales tienen una antigüedad de 30 años y sólo se han renovado parcialmente, pero en el fondo marchan rezagados en comparación con las clínicas o consultorios privados.

2. Es necesario superar esta situación que dificulta una mejor atención a los pacientes que acuden al servicio y que debiera ser mejorada con eventos de actualización y especialización organizados por el Hospital, en alianza estratégica con el Colegio de Odontólogos y las universidades de Lima.

\section{REFERENCIAS BIBLIOGRÁFICAS}

1. Lutz F, Phillips RW, Rouler JF, Sercos JC. In vivo and in vitro of potencial posterior composites, J Dent Res 1984; 63: 914-920.

2. Rueggeberg FA, Caughman WF, Curtis JW, Jr.,m Dawis HC. Factors affecting cure at depth within light-activated resin composites. Am Jden 1993; 6: 91-95.

3. Kilian RJ. The application of photochemistry to dental material, in Gebelein CG, Koblirz FK. Biomedical and dental application of polymers. New York: Pleniun Press; 1981: 411-416.

4. Pearson CJ, Longmn CM Water Sorption and solubility of resin based materials following inadecuate polymerization by a visible-light curing system. J Oral Rehab 1989; 16: 57-61.

5. Curtis JW, Rueggeberg FA; et al. Eficiencia del fotocurado con punta turbo. J Clínica en Odont 1996/1997; 12(3): 19-27.

6. Pires JA. Effects of curing tip distance on light intensity and compose resin micro hardness. Quintessence Int. 1993, 24 (7): 517-521.
7. Marra LM: An historical review of full coverage of the natural dentition.NY State Dent J: 36: 147. 1970.

8. Smith, D: Spreafico: Adhesivo metal free restorations. Quintessence International Chicago 1997.

9. Bowen, R.L: Dental Filling Material comprising vinil silane trated fused silica and a binder consisting of the reaction product of bisphenol and glycitil acrilate. US. Patent No 3,066, 112, 1962.

10. Ott, G Composites filling materials-ivoclarvivadent. Report. No.5. 1990: 3-5.

11. Ferracane, JL. Current trnds in Dental Composites. Crit Rev. Oral Biol Med. 1995; 6(4): 302-318.

12. Phillips, RW. Composite Resin System (comments) Annals Academy of Medicine. 1996; 15(3): 438.

13. Nadarajah, V.et al. Local inflamatory effects of Composte Resins. Compendium. 1997; 18 (4): 367-374.

14. Bayne, S.C et al. Update on dental composite restorations. J. Amer. Dent. Assoc. 1994; 125: 687-701.

15. Leinfelder, K.F Posterior Composites. State of the art, clinical applications. Dent. N. Amer. 1993; 37(3): 411-418.

16. Adep Report, Alberts, HF, editor. Direct Composite Restoratives. 1991; 2(4):53-65.

17. R. W. Bryant. Resinas de composite 200; IX. 95-97

18. Chung (1990). The relationship between composition and properties of posterior resin composites. Journal Dental Research. 69 (3): $852-856$.

19. Macorra Garcia and others (2000) Polymerization contraction of composite resin restorative materials. Sociedad Española de Odontologia Conservadora. Volumen 2. No 1: $24-35$. 
20. Buschlicher and others (1997) Effect of composite type, light intensity, configuration and lase polymerization. American Journal Dental 10: 88 - 96.

21. Versluis and Others (1998). Do Dental composite resins always shrink towards the light? Journal Dental Research. 77(6): 1435 -1445 .

22. Asmussen E. (1998) Direction of shrinkage of light curing resin composites. Journal Dental Research. 77 Abstr. No 212.
23. Hansen (1982) Visible light cured composite resins. Scandinava. Journal Dental research 90: $329-335$.

24. Aster D, Feilzer, and Davidson. (1995) Tensile strength of thin resin composite layers as a funtion of layer - thickness. Journal Dental Research. 74 (11): 1745 - 1748.

25. Kovan, P; Kurschnev, R. Efect of secuntial vasos continous irradiation of a light-cured resin composite on shin fage, viscosity, adhesion, and degree of polymerization. Siencia journal of dentistry. February 1998; 11(1): 17-22 